\title{
ESR Studies of Molecular Motion of Spin Labelled Poly(methyl methacrylate)
}

\author{
Masaru Shiotani and Junkichi Sohma \\ Faculty of Engineering, Hokkaido University, \\ Sapporo 060, Japan.
}

(Received January 7, 1977)

\begin{abstract}
ERS studies of the nitroxide spin-labelled poly(methyl methacrylate) (PMMA) in the solid phase were carried out to obtain information about molecular motion of the order of nanosecond. The amorphous line-shape was partially averaged in the temperature range between 90 and $140^{\circ} \mathrm{C}$, above which the spectra appeared isotropically and narrowed gradually. Three different methods were used to obtain the correlation times from the observed spectra over the wide range of temperature. The comparison of the correlation times determined by different analysis from identical data was discussed. The correlation times and the activation energies, which were obtained by different analyses from the identical data, were found to be nearly equal one to another. Although the mechanism of the fast mode of the motion of PMMA molecules in the solid phase is not yet well understood, it is plausible to assume that the motion resulting in the partial averaging is related to the rotation of the ester side chain.

KEY WORDS ESR / Molecular Motion / PMMA / Spin-Label / Analysis of Partial Average /
\end{abstract}

Since ESR parameters, such as the $g$ factor and the hyperfine (hf) coupling constant, of a free radical are tensors, an ESR spectrum determined by these parameters depends on the orientation of the responsible radical with reference to a space-fixed direction, such as a static magnetic field. When radicals are immobilized in an amorphous matrix, the observed ESR spectrum appears as a characteristic line-shape, the so-called "amorphous pattern,",1,2 in the case of random distribution. When molecular motion of the radical in solid is activated these anisotropic parameters are averaged out if the motion is sufficiently rapid, and the observed spectrum is "isotropic" just as it is from a radical in a liquid of low viscosity. In an intermediate case, which commonly occurs in polymers, the anisotropy is partially averaged; such partial averaging was reported for a solid polymer. ${ }^{3}$ In the case of rapid molecular motion, the anisotropy of the ESR parameters still contributes to the line-widths of peaks through the spin-spin relaxation resulting from the fluctuation of the exact resonance frequencies. ${ }^{4}$ The correlation time $\tau_{\mathrm{c}}$ of the fluctuation can be quantitatively determined from the observed linewidth, $1 / T_{2}{ }^{4}$ Therefore, it is possible to obtain information on molecular motion by analysis of either the changes in line-shape of an ESR spectrum, which are directly influenced by the partial averaging of the $\mathrm{hf}$ coupling tensor, or the changes of the line-width, $1 / T_{2}$, of each peak of hf lines. Since the order of magnitude of anisotropy in a hf coupling is about $10^{8} \mathrm{~Hz}$, the time scale of molecular motion required to average the anisotropy is one shorter than $10^{-8} \mathrm{sec}$. Experimental techniques, used commonly to study molecular motions of polymers, are insensitive to such rapid molecular motion. A quantitative analysis of the variation of ESR spectrum from a polymer radical is of interest, because one may obtain quantitative information of molecular motion in a very rapid mode, which has not yet been studied in detail in polymers.

Several reports on ESR studies on the molecular motion of several polymers were published in recent years. Rabold successfully applied the spin-probe method to several synthetic 
polymers, such as polyethylene, polypropylene, polystyrene and poly(vinyl chloride), in order to study the molecular motion of the polymers. ${ }^{5,6}$ Bullock and his associates carried out extensive studies on spin-labelled polystyrene, investigating quantitatively the segmental motions of polystyrene in various solvents as well as in solid phase. ${ }^{8-11}$ Kusumoto and his collaborators employed the spin-probe technique to detect defects in the polyethylene crystal ${ }^{12}$ and segmental diffusion in the rubbers. ${ }^{13}$ Veksli and Miller published ESR studies of molecular motion of polystyrene ${ }^{14}$ and other polymers. ${ }^{15}$ Recently Tsutsumi; Hikichi, and Kaneko reported spinprobe studies of poly( $\gamma$-benzyl L-glutamate).$^{16}$ ESR spectra of the spin-labelled poly(methyl methacrylate) (PMMA) in solid phase were observed and analyzed by Miller and Veksli $^{15}$ as well as by the present authors in their preliminary report. ${ }^{17}$ Bullock, et al., recently published ESR studies of the spin-labelled PMMA and other acrylate polymers in liquid solutions. ${ }^{18}$ It seems to us that more detailed research is still needed to obtain quantitative information on the rapid molecular motion of PMMA in the solid phase by using the spin-labelled technique. This can be done by a quantitative analysis of an observed ESR spectrum. However, a method for analysis of a partially averaged line-shape has not been definitely established, while the Kivelson theory ${ }^{4}$ is widely used to estimate a correlation time from a completely averaged spectrum. ${ }^{5,7,15}$ Freed developed a sophisticated theory, ${ }^{19}$ by which one could simulate a spectrum to an observed one under condition of the partial average, and could determine a rotational correlation time from the observed spectrum. Freed's theory is too rigorous to permit of easy application to polymers. Based on his own exact theory. Freed, et al.,$^{20,21}$ proposed a simple analytical expression, which is very convenient to estimate the correlation times. Moriuchi and Sohma presented a theoretical approach to the quantitative analysis of the partially averaged spectrum. ${ }^{22}$ In this work, ESR spectra of the spin-labelled PMMA were measured over a wide range of temperature and the rotational correlation times were estimated by making use of the several methods of the analysis for comparison. The results will be discussed in terms of the modes of the molecular motion in PMMA.

\section{EXPERIMENTAL}

Sample

The used sample, PMMA having the covalently attached nitroxide at the ends of the ester side chains (Figure 1), was prepared in the procedure reported by Ohnishi and Itoh. ${ }^{23}$ The nitroxide radical 2,2,6,6-tetramethyl-4-hydroxylpiperidine-1-oxyl was made from 2,2,6,6-tetramethyl piperidinol by using the conventional method. ${ }^{24}$ 4-methylacryloxyl-2,2,6,6-tetramethylpiperidine (A in Figure 1) was employed as the precursor monomer, which was prepared from methacrylolchloride and the corresponding hydroxylpiperidine by a method similar to that published by Kurosaki, et al. ${ }^{25}$ MMA monomers were copolymerized with the precursor monomers, which were spin-labelled with the nitroxides as the side-chains, by using the conventional method of anionic polymerization. The concentration of the spin-labelled monomer was controlled by the mole ratio of monomers of the normal MMA and of the spin-labelled one. The mole ratio of the sample used in this work was taken at $100: 1$. The molecular weight determined by the vapor pressure method was approximately 7800 .

\section{ESR Spectrometer}

A JEOL PE-X spectrometer was used with $100 \mathrm{kHz}$ modulation, and the temperature con-

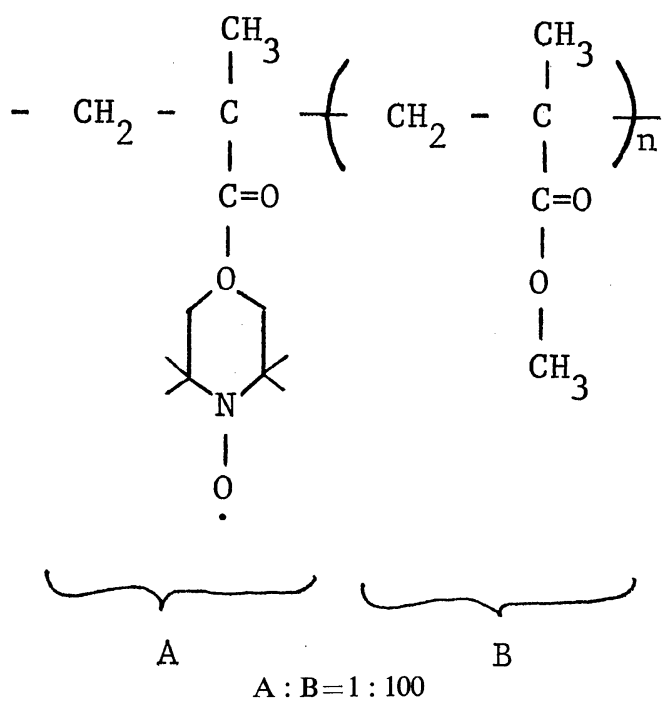

Figure 1. Spin-labelled PMMA with nitroxide at the end of the side chain. 
trol unit attached to the spectrometer was used for temperature variation experiments.

\section{RESULTS}

The examples of ESR spectra observed from the spin-labelled PMMA in a solid phase are shown in Figure 2 for the various temperatures under observation. Apparently the line shape changes gradually to $175^{\circ} \mathrm{C}$, and the pattern observed at $196^{\circ} \mathrm{C}$ is similar to the spectrum of a nitroxide radical in a viscous liquid phase. ${ }^{18,23,26}$ This fact means that the molecular motion of the spin-labelled sites of PMMA at this temperature is as rapid as in the liquid phase. The peak to peak separations of the two outermost extrema (the extrema separations), marked with the arrows in Figure 2, are plotted against the observed temperatures in Figure 3. This separation stays almost unvaried up to $c a .80^{\circ} \mathrm{C}$

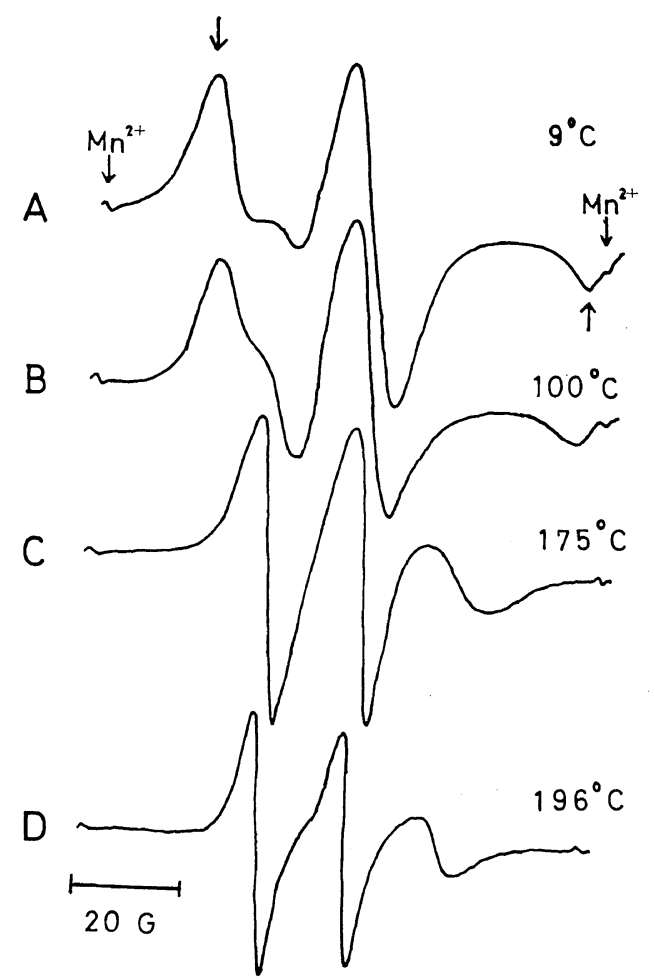

Figure 2. Temperature variation of the observed ESR spectra. The separation between the bold arrows and the corresponding one were taken as the extreme separations. Temperatures in the figure are observation temperature.

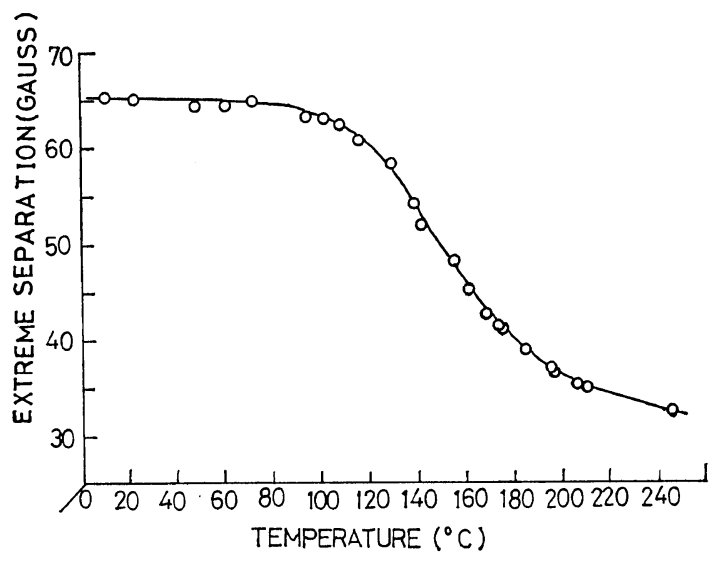

Figure 3. Temperature variation of the extreme separation.

from $-196^{\circ} \mathrm{C}$ and decreases markedly from $100^{\circ} \mathrm{C}$ to the highest temperature in the experiments. The apparent invariance of the extrema separation below $80^{\circ} \mathrm{C}$ indicates that the molecular motion resulting in the partial averaging is sufficiently frozen out in this temperature range. Temperature variations of each peak, $h\left(M_{\mathrm{I}}\right)$, of the observed triplet spectra are shown in Figure 4. There is a break at about $150^{\circ} \mathrm{C}$ in each curve and the peak intensity reaches a maximum at $190^{\circ} \mathrm{C}$, from which the nitroxide

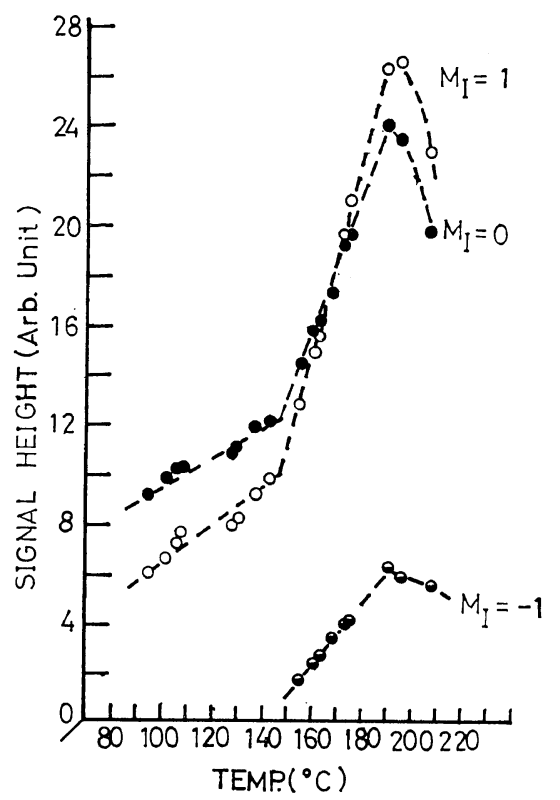

Figure 4. Temperature variation of the peakheights of each line of the nitrogen triplet. 
radicals become unstable and begin to decay. The temperature of the breaks viz. $150^{\circ} \mathrm{C}$, coincides approximately with the temperature from which the ESR spectra appeared isotropically, such as $\mathrm{C}$ in Figure 2. Beyond this temperature the spectrum changes in neither the line shape nor in the extrema separation but rather mainly in the line-width, as shown in Figure 2, and the peaks become narrower as the temperature increases. Thus, the steep increase above this temperature in Figure 4 originates from the narrowing of the line. These results indicate that the polymer molecules are so freely mobile above $150^{\circ} \mathrm{C}$ that the anisotropy of the hf coupling is sufficiently averaged out. This condition is called the "complete average." Below this temperature the molecular motion of the polymers contributes to the partial averaging of the anisotropy of the hf coupling.

\section{THEORIES FOR AN ANALYSIS OF THE SPCTRUM}

The Theory for the Partial Average of the $h f$ Anisotropy

This theory for the partial average of the hf anisotropy was developed by Moriuchi and Sohma ${ }^{22}$ on the basis of the following assumptions:

i) The free radicals are randomly oriented in the space.

ii) The line-shape is mainly determined by the anisotropy of the hf coupling and the linewidth.

iii) The Brownian motion of the free radicals in the system is independent of the magnetic interaction between these radicals and their neighbors or of the external field.

The spin Hamiltonian is divided into two parts,

$$
\mathscr{H}=\mathscr{H}_{0}+\mathscr{H}_{1}(t)
$$

$\mathscr{C}_{0}$, which consists of the isotropic parts, is time-independent and is described as

$$
\mathscr{C}_{0}=g_{0} \beta H \cdot S+A_{0} I \cdot S
$$

where $H, S, I$, and $\beta$, represent the external magnetic field, electron and nuclear spin operators and the Bohr magneton, respectively, with $g_{0}$ and $A_{0}$ standing for the isotropic $g$ factor and hf coupling constant. The other part of the Hamiltonian, $\mathscr{H}_{1}(t)$, is a contribution from the anisotropic part and a fluctuating perturbation, which is averaged out in the case of sufficiently rapid molecular motion. This time-dependent Hamiltonian is given by the following equation

$$
\begin{aligned}
\mathscr{H}_{1}(t)=I \cdot \tilde{A} \cdot S & \\
\tilde{A} & =\left(\begin{array}{ccc}
A_{x} & 0 & 0 \\
0 & A_{y} & 0 \\
0 & 0 & A_{z}
\end{array}\right)
\end{aligned}
$$

in which $A_{x}, A_{y}, A_{z}$ are the principal components of the hf tensor in reference to molecular coordinates. Since only the rotational motion of a radical is effective in averaging the anisotropic hf coupling, an equation of angular diffusion governs the probability density, $P(\alpha, \beta, t)$, to find a free radical at time $t$, an orientation described by Eulerian angles, $\alpha, \beta$, as follows:

$$
\frac{\partial P(\alpha \beta t)}{\partial t}=D \Delta_{\alpha \beta} P
$$

in which $D$ is the angular diffusion coefficient. The eq 5 can be solved with the initial condition and a solution is expressed as a spherical harmonic function,

$$
\begin{aligned}
& P\left(\alpha_{0}, \beta_{0}, t, \alpha, \beta\right) \\
& \quad=\sum_{l} \exp \left[-l(l+1) t / 6 \tau_{\mathbf{c}}\right] Y_{l}{ }^{m}\left(\alpha_{0}, \beta_{0}\right) \cdot Y_{l}{ }^{m}(\alpha, \beta)
\end{aligned}
$$

in which, $\alpha_{0}$ and $\beta_{0}$ are the initial values of $\alpha$ and $\beta$, respectively, and $\tau_{\mathrm{c}}$ is the rotational correlation time. The dipole-dipole interaction, from which the anisotropic hf coupling $\tilde{A}$ originates, is expressed with spherical harmonics. ${ }^{22,27}$ For example,

$$
\begin{aligned}
\mathscr{H}_{1}(t)= & \sum_{i} C\left\langle\psi\left|r_{i}{ }^{-3} Y_{2}{ }^{0}\left(\theta_{i}, \phi_{i}\right)\right| \psi\right\rangle \\
& \times\left[I_{i z} S_{z}-\frac{1}{4}\left(I_{i+} S_{-}+I_{i-} S_{+}\right)\right]
\end{aligned}
$$

in which $Y_{2}^{0}$ is a spherical harmonic function with $l=2, m=0, r_{i}, \theta_{i}$, and $\phi_{i}$ are the spherical coordinates of the nucleus $i$, and $C$ is a constant. Consequently, the average of $\mathscr{H}_{1}(t)$ due to the random rotational motion is represented with $\left\langle Y_{2}{ }^{0}\right\rangle_{\mathrm{AV}}$, the latter being obtained by use of the probability density $P(\alpha, \beta)$ as follows.

$$
\left\langle\mathscr{H}_{1}\right\rangle_{\mathrm{Av}}=\text { const. }\left\langle Y_{2}{ }^{0}\right\rangle_{\mathrm{Av}}
$$

and

$$
\left\langle Y_{2}{ }^{0}\right\rangle_{\mathrm{AV}}=\iint P Y_{2}{ }^{0}(\alpha, \beta) \sin \beta \mathrm{d} \beta \mathrm{d} \alpha
$$

with $\left\langle Y_{l}{ }^{m}\right\rangle_{\mathrm{Av}}$ being also described by the Fourier 
expansion as follows:

$$
\left\langle Y_{l}{ }^{m}\right\rangle_{\mathrm{AV}}=\int_{-\infty}^{\infty} a\left(\alpha_{0}, \beta_{0}, \nu\right) \exp (i 2 \pi \nu \tau) \mathrm{d} \nu
$$

The integration will be performed with the approximation that the integral limits are taken within the line-width, $\pm 1 / T_{2}{ }^{\prime}$ following Bleombergen, Purcell, and Pound, ${ }^{28}$ since the effective interval in the frequency domain is that of the absorption line. Then,

$$
\begin{aligned}
\left\langle\mathscr{H}_{1}\right\rangle_{\mathrm{A} \nabla} & =C^{\prime} \int_{-1 / \pi T_{2}{ }^{\prime}}^{1 / \pi T_{2}{ }^{\prime}} a\left(\alpha_{0} \beta_{0} \nu\right) \exp (i 2 \pi \nu \tau) \mathrm{d} \nu \\
& =\mathscr{H}_{1}(0) \frac{2}{\pi} \tan ^{-1}\left(\tau_{\mathrm{c}} / T_{2}{ }^{\prime}\right)
\end{aligned}
$$

in which $T_{2}{ }^{\prime}$ is the effective transversal relaxation time determined from the observed linewidth.

Consequently

$$
\begin{aligned}
\langle\mathscr{H}\rangle_{\mathrm{Av}}= & \mathscr{H}_{0}+\left\langle\mathscr{H}_{1}\right\rangle_{\mathrm{A} \nabla} \\
= & g_{0} \beta H S+\left\langle A_{x}{ }^{\prime}\right\rangle_{\mathrm{A} \nabla} I_{x} S_{x}+\left\langle A_{y}{ }^{\prime}\right\rangle_{\mathrm{A} \nabla} I_{y} S_{y} \\
& +\left\langle A_{z}{ }^{\prime}\right\rangle_{\mathrm{A} \nabla} I_{z} S_{z}
\end{aligned}
$$

in which

$$
\begin{aligned}
& \left\langle A_{x}{ }^{\prime}\right\rangle_{\mathrm{A} \nabla}=A_{0}+A_{x}(0)(2 / \pi) \tan ^{-1}\left(\tau_{\mathrm{c}} / T_{2}{ }^{\prime}\right) \\
& \left\langle A_{y}{ }^{\prime}\right\rangle_{\mathrm{AV}}=A_{0}+A_{y}(0)(2 / \pi) \tan ^{-1}\left(\tau_{\mathrm{c}} / T_{2}{ }^{\prime}\right) \\
& \left\langle A_{z}{ }^{\prime}\right\rangle_{\mathrm{Av}}=A_{0}+A_{z}(0)(2 / \pi) \tan ^{-1}\left(\tau_{\mathrm{c}} / T_{2}{ }^{\prime}\right)
\end{aligned}
$$

and

$$
A_{0}=\frac{1}{3}\left\{A_{x}{ }^{\prime}(0)+A_{y}{ }^{\prime}(0)+A_{z}{ }^{\prime}(0)\right\}
$$

in which $A_{i}{ }^{\prime}(0)$ is the observed principal value of the hf coupling tensor in the completely frozen state. The details of the derivation have been described in another paper. ${ }^{22}$

The observed values of the extrema separation at the temperatures, which are shown in Figure 3, correspond to $2\left\langle A_{z}{ }^{\prime}\right\rangle_{\mathrm{AV}}$ and are inserted into the third equation of eq 13 . The line-width $1 / T_{2}{ }^{\prime}$ can be determined by a computer simulation utilizing the Maruani-Lefebvre program. $^{29}$ The isotropic coupling constant $A_{0}$ is experimentally determined from an isotropic spectrum, which is observed in the complete average condition. One can estimate the correlation time $\tau_{\mathrm{c}}$ at each temperature level from these data and from the equation.

\section{Freed's Theory}

Another theory, which is applicable to the partial averaging, has been developed by Freed and his collaborators. The partial averaging is measured by the ratio $S=A_{z}{ }^{\prime} \mid A_{z}{ }^{\prime}(0)$, where $A_{z}{ }^{\prime}$ is one half of the extrema separation in the partial averaged line-shape. The correlation time $\tau_{\mathrm{c}}$ is connected with the temperature dependent parameters $S$ by the following equation.

$$
\tau_{\mathrm{c}}=c(1-S)^{d}
$$

in which $c$ and $d$ are dependent upon the diffusion models adopted:

$$
\begin{array}{rl}
\text { Brownian diffusion } & =5.4 \times 10^{-10} \mathrm{sec} \\
& d=-1.36 \\
\text { "Moderate Jump" diffusion } c & =1.10 \times 10^{-9} \mathrm{sec} \\
& d=-1.01 \\
c & =2.55 \times 10^{-9} \mathrm{sec} \\
d & d=-0.615
\end{array}
$$

In the present case of the spin-labelled PMMA no one it seems knows a criterion for choosing one of the above three models over the others. Thus, three different types of correlation times were calculated on the basis of the three different models from a single observation of the extrema separation.

The Theory for the Estimation of the Correlation Time Pertaining to the Case of Complete Average. The above-mentioned theories are not applicable to the case of the complete average and therefore another theory is needed for estimating the correlation time. Kivelson ${ }^{4}$ developed a theory which is applicable cases of complete average, showing that the correlation time may be determined by the following equation.

$$
\begin{aligned}
\frac{1}{T_{2}\left(M_{\mathrm{I}}\right)}= & \tau_{\mathrm{c}}\left\{\left(6+5 M_{\mathrm{I}}^{2}\right) \frac{b^{2}}{40}+\frac{4}{45}(\Delta \gamma \cdot H)^{2}\right. \\
& \left.-\frac{4}{15} b \cdot \Delta \gamma \cdot M_{\mathrm{I}}\right\}
\end{aligned}
$$

in which $M_{\mathrm{I}}$ is the nuclear magnetic quantum number for $I=1$. And $b=(4 \pi / 3)\left(A_{/ /}-A_{\perp}\right), \Delta \gamma=$ $(\beta / h)\left\{g_{z}-\frac{1}{2}\left(g_{x}+g_{y}\right)\right\}$. Assuming the invariance in the line intensity of a spectrum, the following equation can be derived from eq $16^{7}$

$$
\tau_{\mathrm{c}}=\frac{\sqrt{3} \times 15}{b \cdot \Delta \gamma \cdot H} \cdot \Delta H_{\mathrm{msl}}(0)\left\{\sqrt{\frac{h(0)}{h(-1)}}-\sqrt{\frac{h(0)}{h(1)}}\right\}
$$


in which $h(0)$ and $h( \pm 1)$ are the peak heights of $M_{\mathrm{I}}=0$ and $M_{\mathrm{I}}= \pm 1$, respectively.

Determination of the Correlation Time. In the temperature range of the partial average below $150^{\circ} \mathrm{C}$ eq 13 may be used to estimate the correlation times from the observed separations between the two extrema. In this estimation $T_{2}{ }^{\prime}$ was taken as $1.63 \times 10^{-8} \mathrm{sec}$, which was determined from the line width of the best simulated pattern. In the range of the complete average above $150^{\circ} \mathrm{C}$ eq 17 is applied to estimate the correlation times from the observed peak heights shown in Figure 4. The parameters used in eq 17 were $b=8.1$ Gauss $\left(A_{\| /}=32.0 \mathrm{G}\right.$, $\left.A_{\perp}=7.6 \mathrm{G}\right)$ and $\Delta \gamma=4.22 \times 10^{4} \mathrm{Gauss}^{-1} \cdot \mathrm{sec}^{-1}\left(g_{z}=\right.$ 2.0027, $\left.g_{x}=2.0089, g_{y}=2.0051\right)$. The correlation times obtained by the two different methods are plotted against the inverse of the temperature in Figure 5. As shown in Figure 5, the Arrhenius plot of the correlation times obtained by eq 13 from the experimental results at the temperatures lower than $180^{\circ} \mathrm{C}$ is divided into the three states; the temperature-invariance range, the gradual change and, the sharp change. The constant correlation times below $60^{\circ} \mathrm{C}$ do not mean that the correlation times are independent of temperature but they do mean that the correlation times in the temperature range are too slow to produce even a partial average of the anisotropy of the hf coupling. In the second temperature region the Arrhenius plot shows good linearity, which gives an activation energy of $2.9 \mathrm{kcal} / \mathrm{mol}$. In the third region the activation energy was ob-

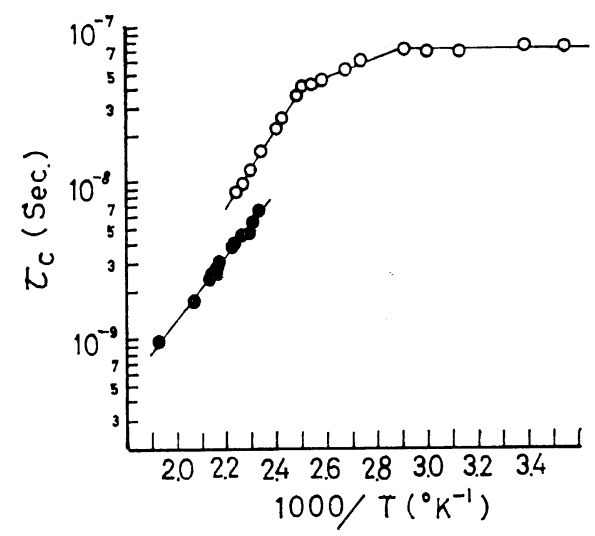

Figure 5. Arrhenius plots of the correlation times obtained by eq 13 and 17 .
Table I. Correlation time estimated by partial average

\begin{tabular}{rcc}
\hline Temp, ${ }^{\circ} \mathrm{C}$ & $\tau_{\mathrm{c}}, 10^{-8} \mathrm{sec}$ & $\Delta E, \mathrm{kcal} / \mathrm{mol}$ \\
\hline 71 & 7.0 & \\
100 & 5.3 & 2.9 \\
128 & 3.6 & \\
\hline 141 & 2.2 & \\
161 & 1.2 & 11.4 \\
175 & 0.82 & \\
\hline
\end{tabular}

tained as $11.4 \mathrm{kcal} / \mathrm{mol}$. The correlation times obtained in the complete average is shown with black circles in Figure 5. The Arrhenius plot is again a straight line, from which the activation energy is estimated as $9.7 \mathrm{kcal} / \mathrm{mol}$. The correlation times obtained and their activation energies are shown in Tables I and II. It is worthy to note that the Arrhenius plot of the correlation times determined from the assumption of the sufficiently rapid molecular motion is approximately parallel to that of the correlation times in the third stage of the partial averaging. Moreover, in the intermediate temperature region, near $150^{\circ} \mathrm{C}$, where either of the two assumptions viz. the partial average or the complete average, can be regarded as a kind of approximation, the correlation times obtained by the two different methods were found to be approximately the same within the error caused by the crude approximations taken in such an intermediate region. These two facts strongly suggest that the two phenomena viz. the temperature variation of the peak heights as well as the partial averaging of the hf coupling tensor in the third stage, originate from the identical mode of motion of the polymer molecule, although it is separately detected from the two different analyses.

Table II. Correlation time estimated by narrowing

\begin{tabular}{ccc}
\hline Temp, ${ }^{\circ} \mathrm{C}$ & $\tau_{c}, 10^{-8} \mathrm{sec}$ & $\Delta E, \mathrm{kcal} / \mathrm{mol}$ \\
\hline 161 & 0.47 & \\
175 & 0.40 & 9.7 \\
190 & 0.26 & \\
210 & 0.17 & \\
\hline
\end{tabular}




\section{DISCUSSION}

Speaking exactly, the polymerization procedure taken in this experiment does not give us any firm ground for the random spin-label in our prepared sample. However, the clear appearance of the triplet spectrum indicates that the local concentration of the spin-label is much less than $10^{-3} \mathrm{M}$, beyond which the line-broadening due to the dipolar interaction changes the triplet to a broad singlet. ${ }^{15}$ Moreover, it was found that the line width increased with the increased concentration of the spin-label. This fact also suggests that the dipole-dipole interaction, which is determined by the inverse cube of the average distance between the neighboring radicals, is proportional to the concentration, and consequently, the random distribution of the spin-label. Therefore, it is safe to assume that no higher local concentration of the nitroxide due to the nonrandom copolymerization is formed in the prepared sample and, the spin-labelled monomers are distributed rather randomly in the PMMA polymer.

The estimation of the correlation time from the observed line width by use of the Kivelson theory is based on the assumption that the observed line width is determined merely by the fluctuation associated with the anisotropy of the ESR parameter. However, there are several factors actually affecting the line width, as for example, the unresolved coupling with the protons in the nitroxide. ${ }^{30}$ It is evident that the couplings with the protons in this system were less than 1 Gauss $^{8,31}$ which is smaller than the line width in this case. It was also found that the line width decreases continuously with the temperature from which the radical decay starts, as shown in Figure 4. This finding seems to support the assumption that the line width observed in this temperature range is mainly determined by the Kivelson mechanism.

The correlation times obtained through eq 15 from the same experimental results are plotted against the inverse of temperatures for comparison with those determined by eq 14 (Figure 6). Three sets of correlation times were obtained depending on the assumed models in the Freed's theory, there being no reason to choose any particular one. The correlation times derived

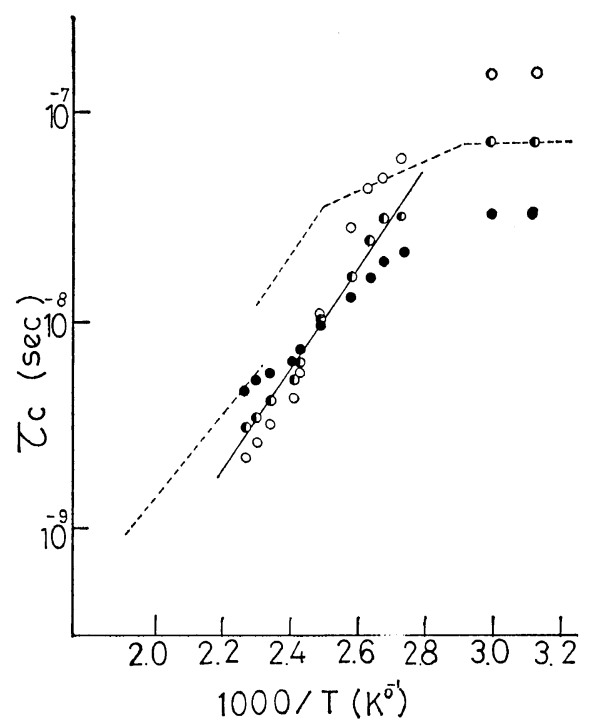

Figure 6. Comparison of the results obtained by eq 13 with those by Freed's equations: $\bigcirc$, Brownian diffusion model; $\mathbf{D}$, moderate jump model; $\mathbf{O}$, large jump diffusion model. The dotted lines are the same Arrhenius plots as those in Figure 5.

from the moderate jump diffusion model are closer to the results determined by the other two methods, eq 13 and 17, as shown in Figure 5, than in the other models. Thus, from the experimental viewpoint one might say that the moderate jump model is the mechanism for the molecular motion of PMMA in that temperature domain near $160^{\circ} \mathrm{C}$.

The discontinuity of the Arrhenius plot at $60^{\circ} \mathrm{C}$, considered to be virtual as mentioned in the previous section, only means that the rate of the molecular motion becomes too slow to average the hf coupling anisotropy. A break in the Arrhenius plot at $128^{\circ} \mathrm{C}$ is taken as a transition in the molecular motion of PMMA, because the activation energy above this transition is apparently different from that in the lower temperature region. The nature of these modes of molecular motion of PMMA is not yet well understood. It is difficult to compare the obtained correlation times with other data, because the nanosecond correlation times are hardly determined by other experimental methods and no other datum in this time range on solid PMMA has been published to the authors' best knowledge. The correlation times determined by 
${ }^{13} \mathrm{C}$-NMR relaxation cover the similar domain of the time scale, ${ }^{32}$ but the application of this technique is just limited to a liquid phase. Recent experiments of excimer emission, ${ }^{33}$ which provides information on the mode of nanosecond molecular motion, are also inapplicable to the solid PMMA. From the spin-lattice relaxation measurement on the solid PMMA, the temperature at which the time scale of the segmental motion of the main chain was of the order of $10^{-7} \mathrm{sec}$, was reported to be $150^{\circ} \mathrm{C}$ or higher. ${ }^{34}$ If the molecular motion responsible for the averaging is ascribed to the same motion, the averaging temperature should shift to a temperature higher than $150^{\circ} \mathrm{C}$ because the faster mode affects the averaging. The observed temperature, however, was $128^{\circ} \mathrm{C}$, lower than even the temperature corresponding to $10^{-7} \mathrm{sec}$. In the dispersion map of PMMA the Arrhenius plot of the easter side chain motion crosses that of the main chain motion at about $100^{\circ} \mathrm{C} .^{35}$ Thus, it is not easy to definitely assign the fast molecular motion at these temperatures even through use of the dispersion map. The activation energy determined in this temperature range as a result of this experiment is different from that of the mainchain motion $(40-150 \mathrm{kcal} / \mathrm{mol})^{36}$ but closer to that of the side chain motion, $19 \mathrm{kcal} / \mathrm{mol}^{37} \mathrm{On}$ the basis of these data and considerations presented above, it is not unreasonable to assume that the molecular motion resulting in the partial averaging is the random rotation of the easter side-chain of PMMA. Other experimental approaches are required to obtain information on the faster mode of the molecular motion in solid PMMA.

Acknowledgment. The authors wish to express their cordial thanks to Professor S. Ohnishi, Faculty of Science, Kyoto University for his kind supply of the spin-labelled PMMA and of the preparation method. The authors would like to express their appreciation to Mr. Kentaro Murakami for his assistance in preparation of figures and in calculation of $\tau_{c}$ by Freed's equations.

\section{REFERENCES}

1. R. H. Sands, Phys. Rev., 99, 1222 (1955).

2. T. K. Kneubuhl, J. Chem. Phys., 33, 1074 (1960).
3. S. Moriuchi, M. Nakamura, S. Shimada, H. Kashiwabara, and J. Sohma, Polymer 11, 630, (1970).

4. D. Kivelson, J. Chem. Phys., 33, 1904 (1960).

5. G. P. Rabold, J. Polym. Sci., 7, 1187 (1969).

6. G. P. Rabold, ibid., 7, 1203 (1969).

7. A. T. Bullock, J. H. Butterworth, and G. G. Cameron, Europ. Polym. J., 7, 445 (1971).

8. A. T. Bullock, G. G. Cameron, and P.M. Smith, Polymer, 13, 89 (1972).

9. A. T. Bullock, G. G. Cameron, and P.M. Smith, Faraday Trans. II J. Chem. Soc., 70, 1202 (1974).

10. A. T. Bullock, G. G. Cameron, and P. M. Smith, J. Polym. Sci., 11, 1263 (1973).

11. A. T. Bullock, G. G. Cameron, and P. M. Smith, J. Phys. Chem., 77, 1635 (1973).

12. N. Kusumoto, M. Yonezawa, and Y. Motozato, Polymer, 15, 793 (1974).

13. N. Kusumoto, S. Sano, N. Zaitsu, and Y. Motozato, ibid., 17, 448 (1976).

14. Z. Veksli and W. G. Miller, Macromolecules, 8, 248 (1975).

15. W. G. Miller and Z. Veksli, Rubber Chem. \& Tech., 48, 1078 (1975).

16. A. Tsutsumi, K. Hikichi and M. Kaneko, Polym. J., 8, 511 (1976).

17. M. Shiotani and J. Sohma, Rept. Prog. Polym. Phys. Jpn., 17, 505 (1974).

18. A. T. Bullock, G. G. Cameron, and V. Krajewski, J. Phys. Chem., 80, 1792 (1976).

19. J. H. Freed in "Electron Spin Relaxation in Liquids," L. T. Muus and F. W. Atkins, Eds., Plenum Press, New York, N.Y., 1972, p 165.

20. J. H. Freed, G. V. Bruno, and C. F. Polnaszek, J. Phys. Chem., 75, 3385 (1971).

21. S. A. Goldman, G. V. Bruno, and J. H. Freed, ibid., 76, 1858 (1972).

22. S. Moriuchi and J. Sohma, Memoir Faculy of Eng., Hokkaido Univ., 13, 335 (1974).

23. I. Itoh and S. Ohnishi, 9th ESR Symposium, Chem. Soc. Jpn., 1970.

24. For example, E. G. Rozantzev, and L. A. Krinitzkaya, Tetrahedron, 21, 491 (1965).

25. T. Kurosaki, K. W. Lee, and M. Okawara, $J$. Polym. Sci., Polym. Chem. Ed., 10, 3295 (1972).

26. O. H. Griffith and H. M. McConnell, Proc. Nat. Acad. Sci., 55, 8 (1966).

27. A. Carrington and A. D. McLachlan, "Introduction to Magnetic Resonance," Harper, 1967, Chapter 7.

28. N. Bleombergen, E. M. Purcell, and R.V. Pound, Phys. Rev., 73, 679 (1948).

29. R. Lefebre and J. Maruani, J. Chem. Phys., 47, 1480 (1965). 


\section{Molecular Motion of Spin Labelled PMMA}

30. G. Poggi and C.S. Johnson, Jr., J. Mag. Res., 3, 436 (1970).

31. K. Murakami, M. Shiotani, and J. Sohma, Rept. Prog. Polym. Phys. Jpn., 19, 445 (1976).

32. A. Allerhand and R. K. Hailstone, J. Chem. Phys., 56, 3718 (1972).

33. Y. Nishijima, Progress in Polymer Science Japan, Onogi, Ed., Kodansha, Tokyo, 6, 199
(1973).

34. W. P. Slichter, J. Polym. Sci. C 33 (1966).

35. W. P. Slichter, "NMR Basis Principles and Progress," Diehl and Kosfeld, Eds., 4, 219 (1971).

36. F. Johnson and J. C. Radon, J. Polym. Sci., Polym. Chem., 1995 (1973).

37. Y. Ishida and K. Yamafuji, Kolloid. Zh., 177, 97 (1961). 